\title{
Bilevel transportation problem in neutrosophic environment
}

\author{
Aakanksha Singh ${ }^{1,2} \cdot$ Ritu Arora ${ }^{3}$ (D) Shalini Arora ${ }^{1}$ \\ Received: 30 June 2021 / Revised: 10 October 2021 / Accepted: 23 November 2021 / \\ Published online: 8 January 2022 \\ (c) The Author(s) under exclusive licence to Sociedade Brasileira de Matemática Aplicada e Computacional 2022
}

\begin{abstract}
In the current times of the predominance of COVID-19, almost all the countries are conducting inoculation drives. Given the market's inability to compute how much to manufacture, how to transport and the frequently changing demand, the cost of safely and timely transporting the vaccines from factory to syringe is currently indeterminate. In this paper, we formulate this situation using a bilevel transportation problem with neutrosophic numbers (BLTP-NN). The problem comes from a vaccine manufacturing company where the vaccine is produced and then transported to different distribution centres from where it is further transported to various health centres for the conduction of their vaccination drive. The authors have tried to perceive this situation from two perspectives by formulating two different problems. The first problem is a bilevel linear fractional transportation problem which aims at minimizing the transportation cost in proportion to per unit maximization of quantity transported. The second problem is a bilevel indefinite quadratic transportation problem which aims at minimizing the transportation cost and depreciation cost. In both problems, cost coefficients are neutrosophic numbers along with availabilities and demands in the constraint set. These formulated bilevel transportation problems in neutrosophic environment are solved using goal programming strategy to arrive at a satisfactory solution. The relevance of this work is to help the decision makers in budgeting their finances related to the transportation by strategic disbursement leading to a smooth administration of vaccination program.
\end{abstract}

Keywords Bilevel programming · Linear fractional transportation problem · Indefinite quadratic transportation problem · Neutrosophic number $\cdot$ Goal programming

Communicated by Graçaliz Pereira Dimuro.

$凶$ Ritu Arora

ritu.arora@keshav.du.ac.in

Aakanksha Singh

aakankshasingh@aryabhattacollege.ac.in

Shalini Arora

shaliniarora@igdtuw.ac.in

1 Department of ASH, Indira Gandhi Delhi Technical University for Women, New Delhi 110006, India

2 Department of Mathematics, Aryabhatta College, University of Delhi, New Delhi, India

3 Department of Mathematics, Keshav Mahavidyalaya, University of Delhi, New Delhi, India 
Mathematics Subject Classification 90B06 · 90C20 - 90C26 · 90C32

\section{Introduction}

In real life situations, one may come across a situation where a decision maker has indeterminate information regarding the parameters of his or her problem. Currently, in the prevalence of the COVID-19 pandemic, leading vaccine making companies are facing a challenge of producing and transporting the vaccines in a cost-efficient manner. Alongside, all the countries need to administer the vaccines in stock before they cross their shelf-life or expiry date.

The journey of a vaccine begins at the vaccine manufacturing unit from where it is transported to stores or distribution centres from where it is further transported to various health centres for administration (see Fig. 1). This journey of a vaccine from factory to syringe is full of hurdles as there are many undecisive, indeterminate factors related to transportation cost, demands and availabilities. There are several factors affecting the transportation cost predominantly being the mode of transport which is a refrigerated, temperature-controlled vehicle and demands continuous surveillance of its temperature during the travel and also at the times of loading and unloading. Proper infrastructure during transport such as insulated containers, thermal pallet covers, protection from sunlight, humidity, using GPS tracker for surveillance (to avoid cargo-theft), cleanliness (to avoid contamination) all add to the indecisive transportation cost. As the quantity of vaccines to be transported is large and usually for a long distance, it might require more than one vehicle or the decision maker may rent additional vehicles which adds up to the transportation cost. In cases of emergency, change of mode of transport may be a necessity. Shipping freight by sea is usually less expensive than by air. The decision maker has to keep an eye on transportation costs for different modes and can make the switch so as to avoid loss from untimely delivery.

Apart from transportation cost, administration of a vaccine or a newly discovered vaccine faces unexpected challenges like hesitancy or fears of side effects, negative news on deaths post vaccination, comparison between two or three types of vaccines, wait-and-watch attitude, new variant of the virus, stockpiling, unusual turnout, expiry and damage during packaging resulting in indecisive supplies and demand (shortage or excess) and contributes to the depreciation cost as well.

Thus, a trade-off needs to be maintained to budget the finances and maintain customer satisfaction. Maintaining continuous supply, forecasting vaccine needs, fewer delays, ade-

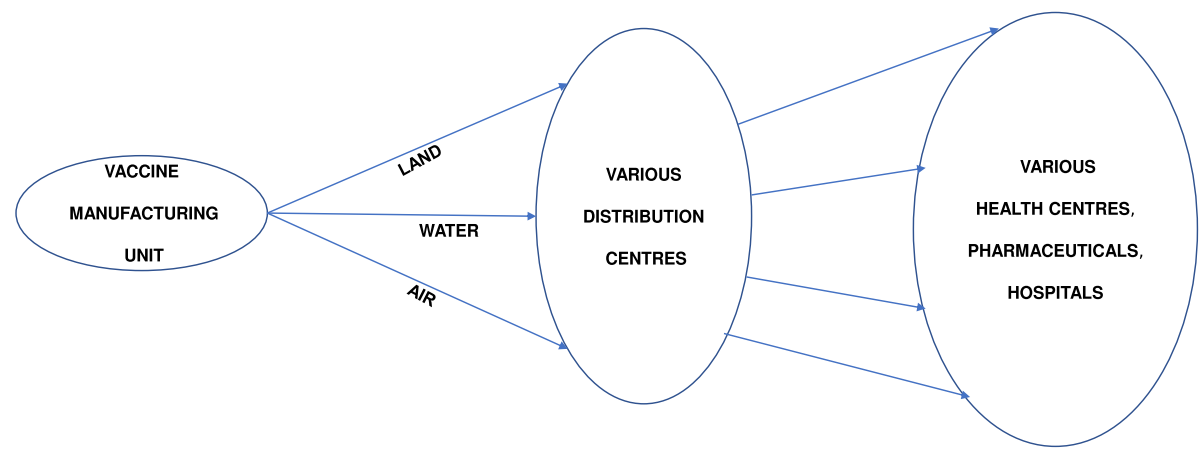

Fig. 1 Transportation route of vaccine through different modes 
quate travel frequency will help in efficient response to the needs of the market else patients will suffer. Looking at the larger picture of public welfare, cooperation and relaxation in decisions amongst the decision makers is crucial.

In this situation where the cost of transport, the quantity to be delivered, availability and demand all are indeterminate we are motivated to use neutrosophic numbers (NNs). This motivates to formulate the above situation as a bilevel transportation problem (BLTP) where the cost coefficients in the objective functions, availabilities and demands all are neutrosophic numbers of the type $P+Q I$ where $P, Q$ are real numbers and $I$ denotes indeterminacy.

For finding the optimal compromise solution, first, we take an appropriate value of $I$ to convert each neutrosophic number in both the objective functions and the constraints into an interval number and transform our problem into a bi-level transportation problem with interval coefficients. Using interval programming technique, the target interval of the objective function of each level is identified and the goal achieving function is formulated.

The objectives of the decision makers are interlinked and potentially conflicting. To deal with this, a plausible relaxation on the respective decision variables is considered so as to avoid decision deadlock. Finally, a goal programming strategy is presented to solve BLTPNN.

\section{Literature review}

Bilevel programming problem (BLPP) is a programming problem with two different hierarchical levels called the upper level and the lower level. Both these levels have their respective decision makers who are interested in optimizing their own benefits. The upper level decision maker is called the leader and the lower level decision maker is called the follower. The leader at the upper level selects his decision vector first. The follower at the lower level then reacts accordingly by choosing his decision vector to optimize its own objective.

The roots of BLPP can be traced in Game Theory by Stackelberg (1952) and in Bracken and McGill (1973) where BLPP appeared as an optimization problem whose constraints themselves contain an optimization problem. Candler and Townsley (1982) proposed the first enumeration algorithm for the linear BLPP where the upper level has no constraints and the lower level problem has unique solution. $K$ th best method for BLPP was introduced by Bialas and Karwan (1982, 1984). Arora and Arora (2011) solved linear-quadratic bilevel programming problem using Karush-Kuhn-Tucker (KKT) conditions. This method is mostly applied to the linear BLPP. A comparative study of fuzzy TOPSIS method and Jaya algorithm for solving bi-level multi-objective linear fractional programming problem was presented by Rizk-Allah and Abo-Sinna (2021).

Depending on the situations arising in the transportation sector different types of transportation problems and in fact blend of transportation problems and BLPP can be seen in the literature. Khandelwal and Puri (2008) solved a bilevel time minimization transportation problem using a polynomial bound algorithm. Arora and Thirwani (2013) solved bilevel capacitated fixed charge transportation problem. Kaushal et al. (2020) considered a BLPP where the upper level problem is a fractional transportation problem and the lower level problem is a fixed charge transportation problem.

Uncertainties in the transportation sector has been the work of many researchers. Multiobjective transportation problem when cost, source and decision parameters are in interval form was discussed by Das et al. (1999). Chinneck and Ramadan (2000) discussed about the best $\&$ the worst optimal solution in interval linear programming problem. Safi and Razmjoo 
(2013) developed solution procedures using two different order relations for interval numbers to solve fixed charge transportation problem with parameters in interval form. Midya and Roy (2017) applied the concept of interval programming to fixed charge transportation problem. Recently, use of rough intervals to model uncertainty in transportation was realised by Garg and Rizk-Allah (2021) for a multi-objective transportation problem and by AnithaKumari et al. (2021) for a solid transportation problem.

The effect of COVID-19 pandemic on transportation sector has been interest of many researchers recently. While the effect of the pandemic on air transport and vice-versa has been extensively discussed by Sun et al. (2021); Narasimha et al. (2021) discussed its effect on Indian seaport transportation \& maritime supply chain.

Smarandache (1998) introduced a novel concept in mathematical philosophy called neutrosophic sets to handle inconsistent, incomplete and indeterminate information where indeterminacy is an independent and a crucial factor in decision making. Following its conception, Smarandache $(2013,2014)$ extended his idea to a neutrosophic number $(\mathrm{NN})$ of the type $\mathrm{N}=P+Q I$ where $P, Q$ are real numbers and $I$ denotes the indeterminacy, such that $I . I$ $=I, 0 . I=0$, and $I / I=$ undefined. Here, indeterminacy is non-numerical or in literal context (Maiti et al. 2019).

There are many papers in the field of optimization which have incorporated NNs in an uncertain environment. Ye (2016) and Mondal et al. (2018) used NNs for group decision making problems. Ye (2018) formed a neutrosophic number linear programming problem in a NN environment followed by an example of production planning problem with answer as neutrosophic numbers. Ye et al. (2018) introduced neutrosophic non-linear function and inequalities and formulated neutrosophic non-linear optimization model for constrained and unconstrained problem and their general solution methods.

Goal programming has also been used to obtain optimal compromise solution while handling conflicting objectives. Maiti et al. (2019) gave a goal programming strategy for multi-level multi-objective linear programming problem with neutrosophic numbers. Pramanik and Dey (2020) formulated multilevel programming with neutrosophic numbers using goal programming supported by a solved example. Pramanik and Dey (2019) discussed bilevel linear programming with NNs using goal programming where objective function at both the levels are linear functions. In this paper, we extend their concept to solve BLTP problem with neutrosophic numbers based on a proposed goal programming strategy. To the best of author's knowledge, a BLTP-NN of the type $P+Q I$ is being formulated for the first time in this paper. A summary of the available literature related to transportation problem is given in Table 1.

The rest of the paper is divided as follows: in Sect. 3 we mention some preliminary definitions. Section 4 gives all the notations to be used in the following text along with the feasibility conditions of a transportation problem. Section 5 deals with the definition of a bilevel linear fractional transportation problem with NNs (BLFTP-NN) followed with its solution methodology. Section 6 gives a numerical illustration of BLFTP-NN. Section 7 deals with the definition of a bilevel indefinite quadratic transportation problem with NNs (BLIQTP-NN) followed with its solution methodology. Section 8 gives a numerical illustration of BLIQTP-NN along with sensitivity analysis. A flowchart depicting the proposed methodology to solve BLTP-NN is given in Sect. 9. Section 10 gives the conclusion and future work. 
Table 1 Summary of literature related to transportation problem

\begin{tabular}{lll}
\hline Author and references & Type of NN & $\begin{array}{l}\text { Type of transportation } \\
\text { problem (TP) }\end{array}$ \\
\hline Singh et al. (2017) & Trapezoidal NN & Standard model \\
Chakraborty et al. (2019) & Pentagonal NN & Standard model \\
Sikkannan and Shanmugavel (2020) & Trapezoidal NN & Standard model \\
Paul et al. (2020) & Trapezoidal NN & Solid transportation problem \\
Saini and Sangal (2020) & Single-valued & Standard model \\
& Trapezoidal NN & \\
Proposed paper & P+QI & Bilevel indefinite quadratic \\
& & TP \& \\
\hline
\end{tabular}

\section{Preliminaries}

Definition 1 Ramadan (1996): Interval number: An interval number on the real line $\mathbf{R}$ is denoted as $T=\left[T^{L}, T^{U}\right]=\left\{\lambda: T^{L} \leq \lambda \leq T^{U}\right\}$, where $T^{L}$ and $T^{U}$ represents the lower (left) and the upper (right) limit of the interval number $\mathrm{T}$ on $\mathbf{R}$.

Definition 2 Ye (2016): Neutrosophic number: A neutrosophic number is represented by $u+\tilde{u} I$ where $u, \tilde{u}$ are real numbers, $u$ is the determinate part, $\tilde{u} I$ is the indeterminate part and $I \in\left[I^{\prime}, I^{\prime \prime}\right]$ denotes indeterminacy. Here, $I^{\prime}$ is the lower (left) limit and $I^{\prime \prime}$ is the upper (right) limit of the indeterminacy.

Thus, if we have $S=u+\tilde{u} I$ as the neutrosophic number and $I \in\left[I^{\prime}, I^{\prime \prime}\right]$ then $S=\left[u+\tilde{u} I^{\prime}, u+\tilde{u} I^{\prime \prime}\right]=\left[u^{\prime}, u^{\prime \prime}\right]$.

For example, suppose a neutrosophic number $S=100+5 I$, then 100 is the determinate part and $5 I$ is the indeterminate part. Now, if we consider $I \in[0,1]$ then $S$ becomes an interval number of the form $[100,105]$. In the same example, if we take $I \in[0,0.6]$ then $S$ becomes an interval number of the form [100,103].

\section{Notations}

A neutrosophic number : $y_{n}=y+\tilde{y} I$ where $I \in\left[I^{\prime}, I^{\prime \prime}\right]$

$M_{L}=\left\{1,2, \ldots, m_{1}\right\}=$ No. of sources of leader's problem

$M_{F}=\left\{m_{1}+1, \ldots, m\right\}=$ No. of sources of follower's problem

$N_{L}=\left\{1,2, \ldots, n_{1}\right\}=$ No. of destinations of leader's problem

$N_{F}=\left\{n_{1}+1, \ldots, n\right\}=$ No. of destinations of follower's problem

$M=M_{L} \cup M_{F}$ : Total no. of sources

$N=N_{L} \cup N_{F}$ : Total no. of destinations

$X_{1}=x_{i j}: i \in M_{L}, j \in N_{L}$ (variables controlled by the leader)

$X_{2}=x_{i j}: i \in M_{F}, j \in N_{F}$ (variables controlled by the follower)

$x_{i j}$ : quantity of the commodity transported from $i^{\text {th }}$ source to $j^{\text {th }}$ destination in one truck (of fixed maximum capacity). Also, $x_{i j} \geq 0 \forall i, j$.

$\left(a_{n}\right)_{i}^{L},\left(a_{n}\right)_{i}^{F}$ : Available supply at $i^{t h}$ source of the leader's and the follower's problem respectively 
$\left(b_{n}\right)_{j}^{L},\left(b_{n}\right)_{j}^{F}$ : Demand at $j^{t h}$ destination of the leader's and the follower's problem respectively

Feasibility conditions:

$$
\sum_{i \in M} a_{i} \geq \sum_{j \in N} b_{j} ; \sum_{i \in M_{L}} a_{i}^{L} \geq \sum_{j \in N_{L}} b_{j}^{L} ; \sum_{i \in M_{F}} a_{i}^{F} \geq \sum_{j \in N_{F}} b_{j}^{F} .
$$

\section{Definition of bilevel linear fractional transportation problem with neutrosophic numbers (BLFTP-NN)}

Mathematically, bilevel linear fractional transportation problem with neutrosophic numbers (BLFTP-NN) is

$$
\left(P_{1}\right) \min _{X_{1}} Z_{1}\left(X_{1}, X_{2}\right)=\frac{\left(c_{1}\right)_{n}^{T} X_{1}+\left(c_{2}\right)_{n}^{T} X_{2}}{\left(k_{1}\right)_{n}^{T} X_{1}+\left(k_{2}\right)_{n}^{T} X_{2}}
$$

subject to

$$
\left.\begin{array}{l}
\sum_{j \in N_{L}} x_{i j} \leq\left(a_{n}\right)_{i}^{L} \forall i \in M_{L} \\
\sum_{i \in M_{L}} x_{i j} \geq\left(b_{n}\right)_{j}^{L} \forall j \in N_{L}
\end{array}\right\}
$$

where $X_{2}$ solves

$$
\left.\left(P_{2}\right) \min _{X_{2}} Z_{2}\left(X_{1}, X_{2}\right)=\frac{\left.\left(e_{1}\right)_{n}^{T} X_{1}+\left(e_{2}\right)\right)_{n}^{T} X_{2}}{\left(l_{1}\right)_{n}^{T} X_{1}+\left(l_{2}\right)_{n}^{T} X_{2}} \quad \text { (for a given } X_{1}\right)
$$

subject to

$$
\left.\begin{array}{l}
\sum_{j \in N_{F}} x_{i j} \leq\left(a_{n}\right)_{i}^{F} \forall i \in M_{F} \\
\sum_{i \in M_{F}} x_{i j} \geq\left(b_{n}\right)_{j}^{F} \forall j \in N_{F}
\end{array}\right\}
$$

where

$$
\begin{aligned}
& \left\{\begin{array}{l}
\left(c_{1}\right)_{n}=\left[\left(c_{n}\right)_{i j}^{L}\right] i \in M_{L}, j \in N_{L} \\
\left(e_{1}\right)_{n}=\left[\left(e_{n}\right)_{i j}^{L}\right] i \in M_{L}, j \in N_{L}
\end{array} \quad:\right. \text { neutrosophic cost parameters of leader's problem } \\
& \left\{\begin{array}{l}
\left(c_{2}\right)_{n}=\left[\left(c_{n}\right)_{i j}^{F}\right] i \in M_{F}, j \in N_{F} \\
\left(e_{2}\right)_{n}=\left[\left(e_{n}\right)_{i j}^{F}\right] i \in M_{F}, j \in N_{F}
\end{array} \quad\right. \text { neutrosophic cost parameters of follower's problem } \\
& \left\{\begin{array}{l}
\left(k_{1}\right)_{n}=\left[\left(k_{n}\right)_{i j}^{L}\right] i \in M_{L}, j \in N_{L} \\
\left(l_{1}\right)_{n}=\left[\left(l_{n}\right)_{i j}^{L}\right] i \in M_{L}, j \in N_{L}
\end{array}\right. \\
& \left\{\begin{array}{l}
\left(k_{2}\right)_{n}=\left[\left(k_{n}\right)_{i j}^{F}\right] i \in M_{F}, j \in N_{F} \\
\left(l_{2}\right)_{n}=\left[\left(l_{n}\right)_{i j}^{F}\right] i \in M_{F}, j \in N_{F}
\end{array}\right. \\
& \begin{array}{l}
\text { and } x_{i j} \geq 0 \forall(i, j) \in M \\
M=M_{L} \cup M_{F}, N=N_{L} \cup N_{F}, \\
M_{L}=\left\{1,2, \ldots, m_{1}\right\}, M_{F}=\left\{m_{1}+1, \ldots, m\right\},
\end{array} \\
& \quad N_{L}=\left\{1,2, \ldots, n_{1}\right\}, N_{F}=\left\{n_{1}+1, \ldots, n\right\} .
\end{aligned}
$$


Also we must have, $\left(X_{1}, X_{2}\right) \in D$ where $D=\left\{\left(X_{1}, X_{2}\right) \in \Phi, X_{2} \in \Psi\left(X_{1}\right),\left(k_{1}\right)_{n}^{T} X_{1}+\right.$ $\left.\left(k_{2}\right)_{n}^{T} X_{2}>0,\left(l_{1}\right)_{n}^{T} X_{1}+\left(l_{2}\right)_{n}^{T} X_{2}>0\right\}$ where,

$\Phi=\left\{\left(X_{1}, X_{2}\right):\left(X_{1}, X_{2}\right)\right\}$ satisfy constraint sets Eqs. 2 and 4 and $\Psi\left(X_{1}\right)=$ $X_{2}: X_{2} \in \operatorname{Argmin} Z_{2}\left(X_{1}, X_{2}\right)$ such that $X_{2}$ satisfies constraint set Eq 4\}.

\subsection{Solution methodology for BLFTP-NN using goal programming}

From the above defined formulation and using our notation of neutrosophic numbers (see Sect. 4), we can re-write the general expressions appearing in Eqs. 1 and 3 as,

$$
\begin{aligned}
\left(y_{1}\right)_{n}^{T} X_{1}+\left(y_{2}\right)_{n}^{T} X_{2} & =\sum_{i=1}^{M} \sum_{j=1}^{N}\left[\left(y_{n}\right)_{i j}\right] x_{i j} \\
& =\sum_{i=1}^{M} \sum_{j=1}^{N}\left[y_{i j}+\tilde{y}_{i j} I\right] x_{i j} \quad \text { where } I \in\left[I^{\prime}, I^{\prime \prime}\right]
\end{aligned}
$$

Thus, the problem (BLFTP-NN) simplifies to

$$
\left(P_{1}\right) \min _{X_{1}} Z_{1}\left(X_{1}, X_{2}\right)=\frac{\sum_{i=1}^{M} \sum_{j=1}^{N}\left[c_{i j}+\tilde{c}_{i j} I\right] x_{i j}}{\sum_{i=1}^{M} \sum_{j=1}^{N}\left[k_{i j}+\tilde{k}_{i j} I\right] x_{i j}}
$$

subject to

$$
\left.\begin{array}{l}
\sum_{j \in N_{L}} x_{i j} \leq\left[a_{i}^{L}+\tilde{a}_{i}^{L} I\right] \quad \forall i \in M_{L}, \\
\sum_{i \in M_{L}} x_{i j} \geq\left[b_{j}^{L}+\tilde{b}_{j}^{L} I\right] \forall j \in N_{L}
\end{array}\right\}
$$

where $X_{2}$ solves

$\left(P_{2}\right) \min _{X_{2}} Z_{2}\left(X_{1}, X_{2}\right)=\frac{\sum_{i=1}^{M} \sum_{j=1}^{N}\left[e_{i j}+\tilde{e}_{i j} I\right] x_{i j}}{\sum_{i=1}^{M} \sum_{j=1}^{N}\left[l_{i j}+\tilde{l}_{i j} I\right] x_{i j}} \quad$ (for a given $X_{1}$ )

subject to

$$
\left.\begin{array}{l}
\sum_{j \in N_{F}} x_{i j} \leq\left[a_{i}^{F}+\tilde{a}_{i}^{F} I\right] \quad \forall i \in M_{F} \\
\sum_{i \in M_{F}} x_{i j} \geq\left[b_{j}^{F}+\tilde{b}_{j}^{F} I\right] \quad \forall j \in N_{F}
\end{array}\right\}
$$

Also, $x_{i j} \geq 0 \forall(i, j) \in M \times N$. Taking $I \in\left[I^{\prime}, I^{\prime \prime}\right]$ and using Sect. 3, we reduce each $\mathrm{NN}$ to an interval number. Therefore, the above system reduces to

$$
\left(P_{1}\right) \min _{X_{1}} Z_{1}\left(X_{1}, X_{2}\right)=\frac{\sum_{i=1}^{M} \sum_{j=1}^{N}\left[c_{i j}^{\prime}, c_{i j}^{\prime \prime}\right] x_{i j}}{\sum_{i=1}^{M} \sum_{j=1}^{N}\left[k_{i j}^{\prime}, k_{i j}^{\prime \prime}\right] x_{i j}}
$$

subject to

$$
\left.\begin{array}{l}
\sum_{j \in N_{L}} x_{i j} \leq\left[a_{i}^{\prime{ }^{L}}, a_{i}^{\prime \prime}\right] \forall i \in M_{L} \\
\sum_{i \in M_{L}} x_{i j} \geq\left[b_{j}^{\prime{ }^{L}}, b_{j}^{\prime \prime}\right] \forall j \in N_{L}
\end{array}\right\}
$$

where $X_{2}$ solves

$$
\left(P_{2}\right) \min _{X_{2}} Z_{2}\left(X_{1}, X_{2}\right)=\frac{\sum_{i=1}^{M} \sum_{j=1}^{N}\left[e_{i j}^{\prime}, e_{i j}^{\prime \prime}\right] x_{i j}}{\sum_{i=1}^{M} \sum_{j=1}^{N}\left[l_{i j}^{\prime}, l_{i j}^{\prime \prime}\right] x_{i j}} \quad \text { (for a given } X_{1} \text { ) }
$$


Table 2 Leader's optimization problem for the best and the worst solution

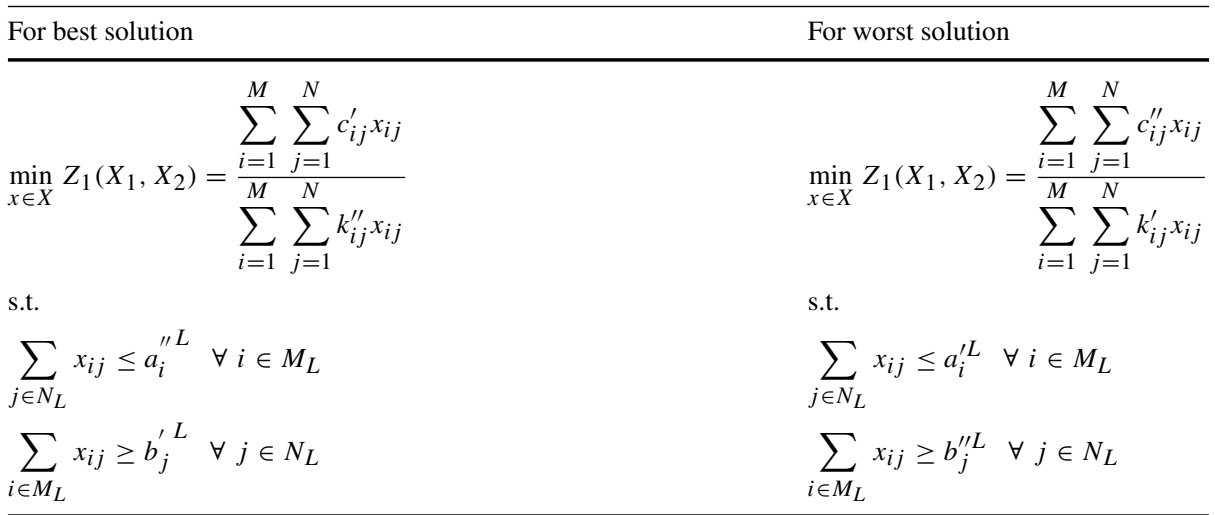

subject to

$$
\left.\begin{array}{l}
\sum_{j \in N_{F}} x_{i j} \leq\left[a_{i}^{\prime F}, a_{i}^{\prime \prime}\right] \forall i \in M_{F}, \\
\sum_{i \in M_{F}} x_{i j} \geq\left[b_{j}^{\prime F}, b_{j}^{\prime \prime}\right] \forall j \in N_{F}
\end{array}\right\}
$$

where $x_{i j} \geq 0 \forall(i, j) \in M \times N$.

At each level, the decision maker would like to know the range of optimal solutions to his problem, i.e. the best optimal (lowest minimum) and the worst optimal (highest minimum) which can be achieved by proper settings of the coefficients (within their intervals) in the objective functions and the constraints (Chinneck and Ramadan 2000). For system constraints, we use the following proposition,

Proposition 1 Shaocheng(1994): Suppose, $\sum_{j=1}^{n}\left[g_{1}^{j}, g_{2}^{j}\right] z_{j} \geq\left[h_{1}, h_{2}\right]$ then $\sum_{j=1}^{n}\left[g_{2}^{j}\right] z_{j} \geq$ $h_{1}$ and $\sum_{j=1}^{n}\left[g_{1}^{j}\right] z_{j} \geq h_{2}$ are the maximum and minimum values range inequalities for the constraint condition, respectively.

Using the above proposition on the constraint sets Eqs. 6 and 7 and taking $\left[g_{1}^{j}, g_{2}^{j}\right]=1$, we obtain the maximum possible feasible region (for best optimal solution) and minimum possible feasible region (for worst optimal solution) for both levels, respectively (Shaocheng 1994; Chinneck and Ramadan 2000). As we are dealing with minimizing an interval fractional objective function, its coefficients are adjusted appropriately for each level. The optimization problem to obtain the best $\&$ the worst solution for both the levels are shown in Tables 2 and 3.

For the $t$ th level decision maker, $t=1,2$, let

$x_{t}^{b}=\left(\left(x_{t}^{b}\right)_{11},\left(x_{t}^{b}\right)_{12}, \ldots\left(x_{t}^{b}\right)_{m_{1} n_{1}},\left(x_{t}^{b}\right)_{m_{1}+1, n_{1}+1}, \ldots\left(x_{t}^{b}\right)_{M N}\right)$ be the individual best optimal solution and $x_{t}^{w}=\left(\left(x_{t}^{w}\right)_{11},\left(x_{t}^{w}\right)_{12}, \ldots\left(x_{t}^{w}\right)_{m_{1} n_{1}},\left(x_{t}^{w}\right)_{m_{1}+1, n_{1}+1}, \ldots\left(x_{t}^{w}\right)_{M N}\right)$ be the individual worst optimal solution.

Also let, $Z_{t}\left(x_{t}^{b}\right)$ to be the corresponding individual best objective function value and $Z_{t}\left(x_{t}^{w}\right)$ to be the corresponding individual worst objective function value. Then, $\left[Z_{t}\left(x_{t}^{b}\right)\right.$, $\left.Z_{t}\left(x_{t}^{w}\right)\right]=\left[\bar{Z}_{t}, \overline{\bar{Z}}_{t}\right]$ is the range of optimal objective function value of the $t^{t h}$ level decision maker in interval form. In a bilevel structured scenario, the individual benefits of each level decision maker are not same and generally conflicting in nature. For obtaining an optimal 
Table 3 Followers's optimization problem for the best and the worst solution

\section{For best solution}

$\min _{x \in X} Z_{2}\left(X_{1}, X_{2}\right)=\frac{\sum_{i=1}^{M} \sum_{j=1}^{N} e_{i j}^{\prime} x_{i j}}{\sum_{i=1}^{M} \sum_{j=1}^{N} l_{i j}^{\prime \prime} x_{i j}}$

s.t.

$$
\begin{aligned}
& \sum_{j \in N_{F}} x_{i j} \leq a_{i}^{\prime F} \quad \forall i \in M_{F} \\
& \sum_{i \in M_{F}} x_{i j} \geq b_{j}^{\prime F} \quad \forall j \in N_{F}
\end{aligned}
$$

For worst solution

$$
\min _{x \in X} Z_{2}\left(X_{1}, X_{2}\right)=\frac{\sum_{i=1}^{M} \sum_{j=1}^{N} e_{i j}^{\prime \prime} x_{i j}}{\sum_{i=1}^{M} \sum_{j=1}^{N} l_{i j}^{\prime} x_{i j}}
$$

s.t.

$$
\begin{aligned}
& \sum_{j \in N_{F}} x_{i j} \leq a_{i}^{\prime F} \quad \forall i \in M_{F} \\
& \sum_{i \in M_{F}} x_{i j} \geq b_{j}^{\prime \prime F} \quad \forall j \in N_{F}
\end{aligned}
$$

compromise solution, an intermediate target interval $\left[Y_{t}^{*}, Y_{t}^{* *}\right]$ is considered for the $t^{\text {th }}$ level decision maker i.e.

$$
\begin{aligned}
& \overline{\bar{Z}}_{t} \geq Y_{t}^{*}, \\
& \bar{Z}_{t} \leq Y_{t}^{* *} .
\end{aligned}
$$

Hence, the goal achievement functions are represented as

$$
\begin{aligned}
& -\overline{\bar{Z}}_{t}+\overline{\bar{D}}_{t}=-Y_{t}^{*}, \\
& \bar{Z}_{t}+\bar{D}_{t}=Y_{t}^{* *}
\end{aligned}
$$

where $\bar{D}_{t}$ and $\overline{\bar{D}}_{t}(t=1,2)$ are deviational variables.

Also, as expected the respective best solutions at each level are not same, so a possible relaxation on the decision variables under the control of each level is considered to avoid decision deadlock.

Again let, $x_{t}^{b}=\left(\left(x_{t}^{b}\right)_{11},\left(x_{t}^{b}\right)_{12}, \ldots,\left(x_{t}^{b}\right)_{m_{1} n_{1}},\left(x_{t}^{b}\right)_{m_{1}+1, n_{1}+1}, \ldots,\left(x_{t}^{b}\right)_{M N}\right)$ be the individual best optimal solution for the $t^{\text {th }}$ level decision maker.

Suppose $\left(x_{1}^{b}\right)_{i j}-\left(l_{1}\right)_{i j} \&\left(x_{1}^{b}\right)_{i j}+\left(u_{1}\right)_{i j} \forall i \in M^{L}, j \in N^{L}$ be the lower and upper bounds of the decision vector provided by the leader where $\left(l_{1}\right)_{i j} \&\left(u_{1}\right)_{i j}, i \in M^{L}, j \in N^{L}$ are the negative and positive tolerance variables which may be different.

Also suppose that $\left(x_{2}^{b}\right)_{i j}-\left(l_{2}\right)_{i j} \&\left(x_{2}^{b}\right)_{i j}+\left(u_{2}\right)_{i j} \forall i \in M^{F}, j \in N^{F}$ be the lower and upper bounds of the decision vector provided by the follower where $\left(l_{2}\right)_{i j} \&\left(u_{2}\right)_{i j}, i \in$ $M^{F}, j \in N^{F}$ are the negative and positive tolerance variables which may be different. Thus, we can write,

$$
\begin{aligned}
& \left(x_{1}^{b}\right)_{i j}-\left(l_{1}\right)_{i j} \leq\left(x_{1}^{b}\right)_{i j} \leq\left(x_{1}^{b}\right)_{i j}+\left(u_{1}\right)_{i j} \forall i \in M^{L}, j \in N^{L}, \\
& \left(x_{2}^{b}\right)_{i j}-\left(l_{2}\right)_{i j} \leq\left(x_{2}^{b}\right)_{i j} \leq\left(x_{2}^{b}\right)_{i j}+\left(u_{2}\right)_{i j} \forall i \in M^{F}, j \in N^{F} .
\end{aligned}
$$

Using, Eqs. 6, 7, Eqs. (8-11), we propose the goal programming model as:

$$
\begin{array}{r}
\operatorname{Min} \sum_{t=1}^{2}\left(\bar{D}_{t}+\overline{\bar{D}}_{t}\right) \\
\text { subject to } \\
\bar{Z}_{t}+\bar{D}_{t}=Y_{t}^{* *},
\end{array}
$$




$$
\begin{array}{r}
-\overline{\bar{Z}}_{t}+\overline{\bar{D}}_{t}=-Y_{t}^{*}, \\
\sum_{j \in N_{L}} x_{i j} \leq a_{i}^{\prime \prime}, \quad \sum_{j \in N_{L}} x_{i j} \geq a_{i}^{\prime{ }^{L}} \forall i \in M_{L}, \\
\sum_{i \in M_{L}} x_{i j} \leq b_{j}^{\prime \prime}, \quad \sum_{i \in M_{L}} x_{i j} \geq b_{j}{ }^{L} \forall j \in N_{L}, \\
\sum_{j \in N_{F}} x_{i j} \leq a_{i}^{\prime \prime}, \quad \sum_{j \in N_{F}} x_{i j} \geq a_{i}{ }^{\prime F} \forall i \in M_{F}, \\
\sum_{i \in M_{F}} x_{i j} \leq b_{j}{ }^{\prime \prime}, \quad \sum_{i \in M_{F}} x_{i j} \geq b_{j}{ }^{F} \forall j \in N_{F}, \\
\left(x_{1}^{b}\right)_{i j}-\left(l_{1}\right)_{i j} \leq\left(x_{1}^{b}\right)_{i j} \leq\left(x_{1}^{b}\right)_{i j}+\left(u_{1}\right)_{i j} \forall i \in M^{L}, j \in N^{L}, \\
\left(x_{2}^{b}\right)_{i j}-\left(l_{2}\right)_{i j} \leq\left(x_{2}^{b}\right)_{i j} \leq\left(x_{2}^{b}\right)_{i j}+\left(u_{2}\right)_{i j} \forall i \in M^{F}, j \in N^{F}, \\
\bar{D}_{t}, \overline{\bar{D}}_{t}, X \geq 0, t=1,2 .
\end{array}
$$

\section{Numerical illustration of BLFTP-NN}

\subsection{Problem definition}

To formulate our problem, we use the following assumptions:

1. Vaccines are transported in insulated containers (of fixed maximum capacity) and trucks are used for transportation.

2. The names and data taken in the numerical illustrations are hypothetical.

3. There is no theft or stockpiling of the vaccine during transportation or at the distribution centres.

Problem: Consider an Indian vaccine manufacturing company Aushadhi with its manufacturing units (MU) located at Ahmedabad, Hyderabad, Bengaluru and Delhi. After manufacturing, it transports the vaccines to its four distribution centres (DC) located at Maharashtra, Tamil Nadu, Rajasthan and Haryana. From these distribution centres, the distributor collects vaccines and further transports them to health centres (HC) in four different regions of each city: North, East, West and South for vaccination drive. Considering the welfare of the people we have assumed that there is no stockpiling or theft of the vaccine during transport or at the intermediate distribution centre. Thus, the demands of the distribution centres at the upper level is the same as their supply for the lower level.

\subsection{Solved numerical (BLFTP-NN)}

In this illustration, the leader is the medical supply company owner who will aim at minimizing the neutrosophic transportation cost in proportion to per unit maximization of quantity transported from manufacturing units to distribution centres. The follower is the distributor whose objective is to minimize the neutrosophic transportation cost in proportion to per unit maximization of quantity transported from distribution centres to different regions of each city.

At the upper level, let the manufacturing units located at Ahmedabad, Hyderabad, Bengaluru and Delhi have $5+4 I, 5+3 I, 5+6 I$ and $5+2 I$ as their respective supply quantity. 
Table 4 Upper level neutrosophic transportation problem

\begin{tabular}{lllll}
\hline MU/DC & Maharashtra & Tamil Nadu & Rajasthan & Haryana \\
\hline Ahmedabad & $1+2 I$ & $4+5 I$ & $6+I$ & $3+2 I \rightarrow\left[c_{i j}+\tilde{c}_{i j} I\right]$ \\
& $4+I$ & $3+I$ & $1+3 I$ & $6+2 I \rightarrow\left[k_{i j}+\tilde{k}_{i j} I\right]$ \\
Hyderabad & $2+I$ & $3+4 I$ & $4+2 I$ & $7+2 I$ \\
& $3+I$ & $1+4 I$ & $2+3 I$ & $3+4 I$ \\
Bengaluru & $9+6 I$ & $6+2 I$ & $1+4 I$ & $9+2 I$ \\
& $5+2 I$ & $2+4 I$ & $4+5 I$ & $2+5 I$ \\
Delhi & $2+3 I$ & $1+5 I$ & $2+3 I$ & $4+2 I$ \\
& $2+6 I$ & $3+4 I$ & $3+2 I$ & $5+I$ \\
\hline
\end{tabular}

Table 5 Lower level neutrosophic transportation problem

\begin{tabular}{lllll}
\hline DC/HC & North region & East region & West region & South region \\
\hline Maharashtra & $4+2 I$ & $5+7 I$ & $2+I$ & $6+2 I \rightarrow\left[e_{i j}+\tilde{e}_{i j} I\right]$ \\
& $5+I$ & $3+I$ & $4+2 I$ & $10+I \rightarrow\left[l_{i j}+\tilde{l}_{i j} I\right]$ \\
Tamil Nadu & $7+5 I$ & $10+I$ & $8+4 I$ & $8+I$ \\
& $2+2 I$ & $3+4 I$ & $6+3 I$ & $1+5 I$ \\
\multirow{2}{*}{ Rajasthan } & $6+9 I$ & $4+4 I$ & $6+3 I$ & $9+2 I$ \\
\multirow{3}{*}{ Haryana } & $2+2 I$ & $3+2 I$ & $1+3 I$ & $3+6 I$ \\
& $8+I$ & $4+3 I$ & $2+4 I$ & $5+4 I$ \\
& $5+I$ & $3+2 I$ & $6+2 I$ & $3+4 I$ \\
\hline
\end{tabular}

Let the four distribution centres located at Maharashtra, Tamil Nadu, Rajasthan and Haryana have $5+2 I, 4+5 I, 6+4 I$ and $5+4 I$ as their respective demands.

At the lower level, let the health centres in four different regions of each city: North, East, West and South have $6+4 I, 6+3 I, 5+4 I$ and $3+4 I$ as their respective demands. We have taken a balanced transportation problem at both the levels with total supply and total demand equal to $20+15 I$.

Tables 4 and 5 represent the neutrosophic transportation cost and neutrosophic number of trucks at upper and lower level, respectively. In each cell $(i, j)$ of both the tables, top entry denotes the neutrosophic transportation cost and bottom entry denotes neutrosophic number of trucks when vaccine is transported from $i$ th source to $j$ th destination. The computing software LINGO 17.0 is used to solve the problem (BLFTP-NN).

The source and demand constraints at upper level are:

$$
\begin{gathered}
\sum_{j=1}^{4} x_{1 j} \leq 5+4 I, \sum_{j=1}^{4} x_{2 j} \leq 5+3 I, \sum_{j=1}^{4} x_{3 j} \leq 5+6 I, \sum_{j=1}^{4} x_{4 j} \leq 5+2 I \\
\sum_{i=1}^{4} x_{i 1} \geq 5+2 I, \sum_{i=1}^{4} x_{i 2} \geq 4+5 I, \sum_{i=1}^{4} x_{i 3} \geq 6+4 I, \sum_{i=1}^{4} x_{i 4} \geq 5+4 I .
\end{gathered}
$$


Table 6 Best upper level optimization problem

\begin{tabular}{llllll}
\hline MU/DC & Maharashtra & Tamil Nadu & Rajasthan & Haryana \\
\hline Ahmedabad & 1 & 4 & 6 & 3 & 9 \\
Hyderabad & 5 & 4 & & & 8 \\
& 2 & 3 & 4 & 7 & \\
Bengaluru & 4 & 5 & 5 & 7 & 11 \\
& 9 & 6 & 1 & 9 & 7 \\
Delhi & 7 & 6 & 9 & 7 & \\
& 2 & 1 & 2 & 4 & \\
Demand & 5 & 7 & 5 & 6 & 5 \\
\hline
\end{tabular}

The source and demand constraints at lower level are:

$$
\begin{gathered}
\sum_{j=1}^{4} x_{1 j} \leq 5+2 I, \sum_{j=1}^{4} x_{2 j} \leq 4+5 I, \sum_{j=1}^{4} x_{3 j} \leq 6+4 I, \sum_{j=1}^{4} x_{4 j} \leq 5+4 I, \\
\sum_{i=1}^{4} x_{i 1} \geq 6+4 I, \sum_{i=1}^{4} x_{i 2} \geq 6+3 I, \sum_{i=1}^{4} x_{i 3} \geq 5+4 I, \sum_{i=1}^{4} x_{i 4} \geq 3+4 I .
\end{gathered}
$$

Let, $X_{1}=\left\{x_{11}, x_{21}, x_{31}, x_{41}\right\}$ be the variables controlled by the leader and $X_{2}=$ $\left\{x_{12}, x_{13}, x_{14}, x_{22}, x_{23}, x_{24}, x_{32}, x_{33}, x_{34}, x_{42}, x_{43}, x_{44}\right\}$ be the variables controlled by the follower.

Taking $\mathrm{I} \in[0,1]$ in the given problem we reduce the BLTP-NN into a BLTP with interval numbers. Tables 6 and 7 are the optimization problems obtained using Table 2 for finding best and the worst upper level solution, respectively. Similarly, we can obtain optimization problem for finding best and the worst lower level solution using Table 3. For finding the best and the worst solution, appropriate dummy source and destination are added to balance the transportation problem. The results are compiled in Table 8 .

Next, we construct goal achievement functions for both the objective functions at both levels according to the obtained results and assign preference bounds on the decision variables.

Let the target level of the leader's objective function be given by $\left[Y_{1}^{*}, Y_{1}^{* *}\right]=[0.3,0.9] \&$ target level of the follower's objective function be given by $\left[Y_{2}^{*}, Y_{2}^{* *}\right]=[0.7,1.1]$. The goal programming model is developed along with the preference bounds on the decision variables and is represented as

$$
\operatorname{Minimize}\left(\bar{D}_{1}+\bar{D}_{2}+\overline{\bar{D}}_{1}+\overline{\bar{D}}_{2}\right)
$$

subject to

$$
\left[\frac{x_{11}+4 x_{12}+6 x_{13}+3 x_{14}+2 x_{21}+3 x_{22}+4 x_{23}+7 x_{24}+9 x_{31}+\ldots}{5 x_{11}+4 x_{12}+4 x_{13}+8 x_{14}+4 x_{21}+5 x_{22}+5 x_{23}+7 x_{24}+7 x_{31}+\cdots}\right.
$$


Table 7 Worst upper level optimization problem

\begin{tabular}{|c|c|c|c|c|c|}
\hline MU/DC & Maharashtra & Tamil Nadu & Rajasthan & Haryana & \\
\hline \multirow[t]{2}{*}{ Ahmedabad } & 3 & 9 & 7 & 5 & \\
\hline & 4 & 3 & 1 & 6 & \\
\hline \multirow[t]{2}{*}{ Hyderabad } & 3 & 7 & 6 & 9 & \\
\hline & 3 & 1 & 2 & 3 & \\
\hline \multirow[t]{2}{*}{ Bengaluru } & 15 & 8 & 5 & 11 & \\
\hline & 5 & 2 & 4 & 2 & \\
\hline \multirow[t]{2}{*}{ Delhi } & 5 & 6 & 5 & 6 & \\
\hline & 2 & 3 & 3 & 5 & \\
\hline Demand & 7 & 9 & 10 & 9 & \\
\hline
\end{tabular}

Table 8 Individual solutions

\begin{tabular}{lll}
\hline Solution & Best & Worst \\
\hline Upper level & $\bar{Z}_{1}=0.2066$ & $\overline{\bar{Z}}_{1}=1.0568$ \\
& at $x_{1}^{b}=$ & at $x_{1}^{w}=$ \\
& $(4,0,0,5,0,0,0,0,0,0,6,0,1,4,0,0)$ & $(1,0,0,4,5,0,0,0,0,0,5,0,0,0,0,5)$ \\
\hline Solution & Best & Worst \\
\hline Lower level & $\bar{Z}_{2}=0.5648$ & $\overline{\bar{Z}}_{2}=1.3606$ \\
& at $x_{2}^{b}=$ & at $x_{2}^{w}=$ \\
& $(6,0,0,1,0,0,0,0,0,4,0,0,0,2,5,2)$ & $(0,0,0,5,0,0,4,0,0,6,0,0,0,0,5,0)$ \\
\hline
\end{tabular}

$$
\begin{gathered}
\left.\frac{\cdots+6 x_{32}+x_{33}+9 x_{34}+2 x_{41}+x_{42}+2 x_{43}+4 x_{44}}{\cdots+6 x_{32}+9 x_{33}+7 x_{34}+8 x_{41}+7 x_{42}+5 x_{43}+6 x_{44}}\right]+\bar{D}_{1}=0.9 \\
{\left[\frac{-3 x_{11}-9 x_{12}-7 x_{13}-5 x_{14}-3 x_{21}-7 x_{22}-6 x_{23}-9 x_{24}-15 x_{31}-\cdots}{4 x_{11}+3 x_{12}+x_{13}+6 x_{14}+3 x_{21}+x_{22}+2 x_{23}+3 x_{24}+5 x_{31}+\cdots}\right.} \\
\left.\frac{\cdots-8 x_{32}-5 x_{33}-11 x_{34}-5 x_{41}-6 x_{42}-5 x_{43}-6 x_{44}}{\cdots+2 x_{32}+4 x_{33}+2 x_{34}+2 x_{41}+3 x_{42}+3 x_{43}+5 x_{44}}\right]+\bar{D}_{1}=-0.3 \\
{\left[\frac{4 x_{11}+5 x_{12}+2 x_{13}+6 x_{14}+7 x_{21}+10 x_{22}+8 x_{23}+8 x_{24}+6 x_{31}+\ldots}{6 x_{11}+4 x_{12}+6 x_{13}+11 x_{14}+4 x_{21}+7 x_{22}+9 x_{23}+6 x_{24}+4 x_{31}+\cdots}\right.} \\
\left.\frac{\cdots+4 x_{32}+6 x_{33}+9 x_{34}+8 x_{41}+4 x_{42}+2 x_{43}+5 x_{44}}{\cdots+5 x_{32}+4 x_{33}+9 x_{34}+6 x_{41}+5 x_{42}+8 x_{43}+7 x_{44}}\right]+\bar{D}_{2}=1.1 \\
{\left[\frac{-6 x_{11}-12 x_{12}-3 x_{13}-8 x_{14}-12 x_{21}-11 x_{22}-12 x_{23}-9 x_{24}-15 x_{31}-\ldots}{5 x_{11}+3 x_{12}+4 x_{13}+10 x_{14}+2 x_{21}+3 x_{22}+6 x_{23}+x_{24}+2 x_{31}+\cdots}\right.}
\end{gathered}
$$


Table 9 Satisfactory solution of BLFTP-NN

\begin{tabular}{llc}
\hline Solution point & Objective values of leader & Objective values of follower \\
\hline$(1,0,0,6,5,0,0,0,0,5,1,0,1,1,5,0)$ & $(0.48026,1.44943)$ & $(0.71511,1.62015)$
\end{tabular}

$$
\begin{gathered}
\left.\frac{\cdots-8 x_{32}-9 x_{33}-11 x_{34}-9 x_{41}-7 x_{42}-6 x_{43}-9 x_{44}}{\cdots+3 x_{32}+x_{33}+3 x_{34}+5 x_{41}+3 x_{42}+6 x_{43}+3 x_{44}}\right]+\overline{\bar{D}}_{2}=-0.7, \\
x_{11}+x_{12}+x_{13}+x_{14} \leq 7, \quad x_{11}+x_{21}+x_{31}+x_{41} \leq 7, \\
x_{21}+x_{22}+x_{23}+x_{24} \leq 8, \quad x_{12}+x_{22}+x_{32}+x_{42} \leq 9, \\
x_{31}+x_{32}+x_{33}+x_{34} \leq 10, \quad x_{13}+x_{23}+x_{33}+x_{43} \leq 9, \\
x_{41}+x_{42}+x_{43}+x_{44} \leq 9, \quad x_{14}+x_{24}+x_{34}+x_{44} \leq 7, \\
x_{11}+x_{21}+x_{31}+x_{41} \geq 6, \quad x_{11}+x_{12}+x_{13}+x_{14} \geq 5, \\
x_{12}+x_{22}+x_{32}+x_{42} \geq 6, \quad x_{21}+x_{22}+x_{23}+x_{24} \geq 5, \\
x_{13}+x_{23}+x_{33}+x_{43} \geq 6, \quad x_{31}+x_{32}+x_{33}+x_{34} \geq 6, \\
x_{14}+x_{24}+x_{34}+x_{44} \geq 5, \quad x_{41}+x_{42}+x_{43}+x_{44} \geq 5,
\end{gathered}
$$

$$
\begin{gathered}
4-3 \leq x_{11} \leq 4+2,0 \leq x_{12} \leq 0+5,0 \leq x_{13} \leq 0+5,1-1 \leq x_{14} \leq 1+7 \\
0 \leq x_{21} \leq 0+6,0 \leq x_{22} \leq 0+5,0 \leq x_{23} \leq 0+1,0 \leq x_{24} \leq 0+2 \\
0 \leq x_{31} \leq 0+6,4-4 \leq x_{32} \leq 4+1,0 \leq x_{33} \leq 0+5,0 \leq x_{34} \leq 0+5 \\
1-0 \leq x_{41} \leq 1+2,2-2 \leq x_{42} \leq 2+2,5-3 \leq x_{43} \leq 5+3,2-2 \leq x_{44} \leq 2+1 \\
x_{i j} \geq 0 \forall i, j ; \bar{D}_{1}, \bar{D}_{2}, \bar{D}_{1}, \overline{\bar{D}}_{2} \geq 0
\end{gathered}
$$

The solution of the proposed goal programming model is shown in Table 9.

\section{Definition of bilevel indefinite quadratic transportation problem with neutrosophic numbers (BLIQTP-NN)}

Mathematically, bilevel indefinite quadratic transportation problem with neutrosophic numbers (BLIQTP-NN) is

$$
\left(P_{1}\right) \min _{X_{1}} Z_{1}\left(X_{1}, X_{2}\right)=\left(\left(f_{1}\right)_{n}^{T} X_{1}+\left(f_{2}\right)_{n}^{T} X_{2}\right)\left(\left(d_{1}\right)_{n}^{T} X_{1}+\left(d_{2}\right)_{n}^{T} X_{2}\right)
$$

subject to

$$
\left.\begin{array}{l}
\sum_{j \in N_{L}} x_{i j} \leq\left(a_{n}\right)_{i}^{L} \forall i \in M_{L} \\
\sum_{i \in M_{L}} x_{i j} \geq\left(b_{n}\right)_{j}^{L} \quad \forall j \in N_{L}
\end{array}\right\}
$$

where $X_{2}$ solves

$\left(P_{2}\right) \min _{X_{2}} Z_{2}\left(X_{1}, X_{2}\right)=\left(\left(p_{1}\right)_{n}^{T} X_{1}+\left(p_{2}\right)_{n}^{T} X_{2}\right)\left(\left(q_{1}\right)_{n}^{T} X_{1}+\left(q_{2}\right)_{n}^{T} X_{2}\right) \quad$ (for a given $\left.X_{1}\right)$

subject to

$$
\left.\begin{array}{l}
\sum_{j \in N_{F}} x_{i j} \leq\left(a_{n}\right)_{i}^{F} \forall i \in M_{F}, \\
\sum_{i \in M_{F}} x_{i j} \geq\left(b_{n}\right)_{j}^{F} \forall j \in N_{F}
\end{array}\right\}
$$

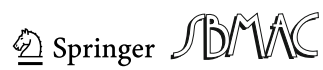


and $x_{i j} \geq 0 \forall(i, j) \in M \times N$.

As before we have, $M=M_{L} \cup M_{F}, N=N_{L} \cup N_{F}$ where $M_{L}=\left\{1,2, \ldots, m_{1}\right\}, M_{F}=$ $\left\{m_{1}+1, \ldots, m\right\}, M_{L}=\left\{1,2, \ldots, n_{1}\right\}, M_{F}=\left\{n_{1}+1, \ldots, n\right\}$.

$$
\begin{gathered}
\left\{\begin{array}{l}
\left(f_{1}\right)_{n}=\left[\left(f_{n}\right)_{i j}^{L}\right] i \in M_{L}, j \in N_{L} \\
\left(p_{1}\right)_{n}=\left[\left(p_{n}\right)_{i j}^{L}\right] i \in M_{L}, j \in N_{L}
\end{array}:\right. \text { neutrosophic transportation cost parameters of leader's problem } \\
\left\{\begin{array}{l}
\left(f_{2}\right)_{n}=\left[\left(f_{n}\right)_{i j}^{F}\right] i \in M_{F}, j \in N_{F} \\
\left(p_{2}\right)_{n}=\left[\left(p_{n}\right)_{i j}^{F}\right] i \in M_{F}, j \in N_{F}
\end{array}:\right. \text { neutrosophic transportation cost parameters of follower's problem } \\
\left\{\begin{array}{l}
\left(d_{1}\right)_{n}=\left[\left(d_{n}\right)_{i j}^{L}\right] i \in M_{L}, j \in N_{L} \\
\left(q_{1}\right)_{n}=\left[\left(q_{n}\right)_{i j}^{L}\right] i \in M_{L}, j \in N_{L}
\end{array}:\right. \text { neutrosophic depreciation cost parameters of leader's problem } \\
\left\{\begin{array}{l}
\left(d_{2}\right)_{n}=\left[\left(d_{n}\right)_{i j}^{F}\right] i \in M_{F}, j \in N_{F} \\
\left(q_{2}\right)_{n}=\left[\left(q_{n}\right)_{i j}^{F}\right] i \in M_{F}, j \in N_{F}
\end{array}:\right. \text { neutrosophic depreciation cost parameters of follower's problem }
\end{gathered}
$$

\subsection{Solution methodology for BLIQTP-NN using goal programming}

We proceed as before by simplifying the expressions in Eqs. 12 and 13 by applying Eqs. 5 (see Sect. 5.1) and obtain the following:

$$
\left(P_{1}\right) \min _{X_{1}} Z_{1}\left(X_{1}, X_{2}\right)=\left(\sum_{i=1}^{M} \sum_{j=1}^{N}\left[f_{i j}+\tilde{f}_{i j} I\right] x_{i j}\right)\left(\sum_{i=1}^{M} \sum_{j=1}^{N}\left[d_{i j}+\tilde{d}_{i j} I\right] x_{i j}\right)
$$

subject to

$$
\left.\begin{array}{c}
\sum_{j \in N_{L}} x_{i j} \leq\left[a_{i}^{L}+\tilde{a}_{i}^{L} I\right] \quad \forall i \in M_{L}, \\
\sum_{i \in M_{L}} x_{i j} \geq\left[b_{j}^{L}+\tilde{b}_{j}^{L} I\right] \quad \forall j \in N_{L}
\end{array}\right\}
$$

where $X_{2}$ solves

$\left(P_{2}\right) \min _{X_{2}} Z_{2}\left(X_{1}, X_{2}\right)=\left(\sum_{i=1}^{M} \sum_{j=1}^{N}\left[p_{i j}+\tilde{p}_{i j} I\right] x_{i j}\right)\left(\sum_{i=1}^{M} \sum_{j=1}^{N}\left[q_{i j}+\tilde{q}_{i j} I\right] x_{i j}\right) \quad$ (for a given $\left.X_{1}\right)$ subject to

$$
\left.\begin{array}{l}
\sum_{j \in N_{F}} x_{i j} \leq\left[a_{i}^{F}+\tilde{a}_{i}^{F} I\right] \quad \forall i \in M_{F} \\
\sum_{i \in M_{F}} x_{i j} \geq\left[b_{j}^{F}+\tilde{b}_{j}^{F} I\right] \forall j \in N_{F}
\end{array}\right\}
$$

with $x_{i j} \geq 0 \forall(i, j) \in M \times N$.

Taking $I \in\left[I^{\prime}, I^{\prime \prime}\right]$ and using Sect. 3 , we reduce each NN to an interval number. Therefore, the above system reduces to

$$
\left(P_{1}\right) \min _{X_{1}} Z_{1}\left(X_{1}, X_{2}\right)=\left(\sum_{i=1}^{M} \sum_{j=1}^{N}\left[f_{i j}^{\prime}, f_{i j}^{\prime \prime}\right] x_{i j}\right)\left(\sum_{i=1}^{M} \sum_{j=1}^{N}\left[d_{i j}^{\prime}, d_{i j}^{\prime \prime}\right] x_{i j}\right)
$$

subject to

$$
\left.\begin{array}{l}
\sum_{j \in N_{L}} x_{i j} \leq\left[a_{i}^{\prime{ }^{L}}, a_{i}^{\prime \prime}{ }^{L}\right] \forall i \in M_{L} \\
\sum_{i \in M_{L}} x_{i j} \geq\left[b_{j}^{\prime}{ }^{L}, b_{j}^{\prime \prime}\right] \forall j \in N_{L}
\end{array}\right\}
$$


Table 10 Leader's optimization problem for the best and the worst solution

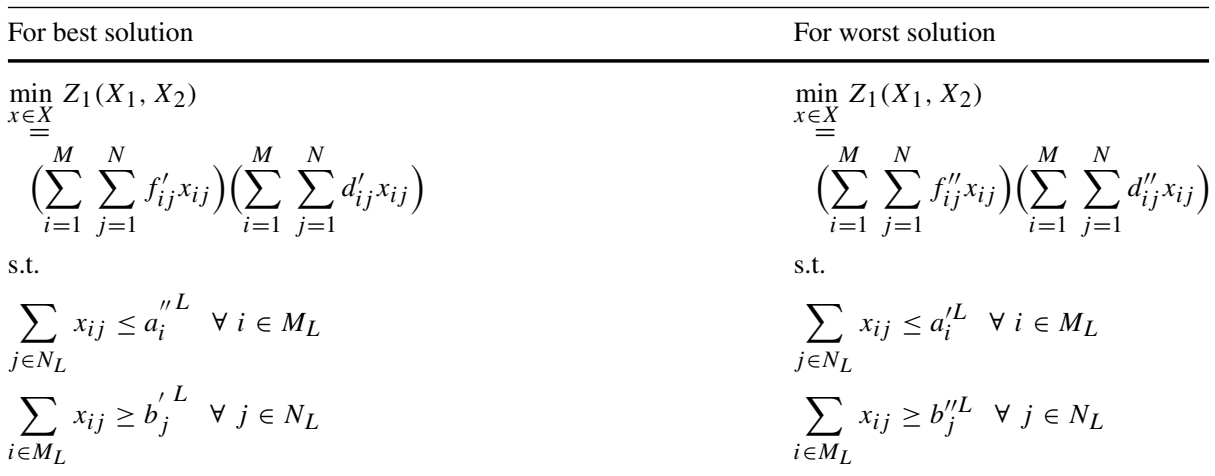

Table 11 Followers's optimization problem for the best and the worst solution

\begin{tabular}{|c|c|}
\hline For best solution & For worst solution \\
\hline $\min _{x \in X} Z_{2}\left(X_{1}, X_{2}\right)$ & $\min _{x \in X} Z_{2}\left(X_{1}, X_{2}\right)$ \\
\hline$\left(\sum_{i=1}^{M} \sum_{j=1}^{N} p_{i j}^{\prime} x_{i j}\right)\left(\sum_{i=1}^{M} \sum_{j=1}^{N}\right.$ & $\left(\sum_{i=1}^{M} \sum_{j=1}^{N} p_{i j}^{\prime \prime} x_{i j}\right)\left(\sum_{i=1}^{M} \sum_{j=1}^{N}\right.$ \\
\hline s.t. & s.t. \\
\hline$\sum_{j \in N_{F}} x_{i j} \leq a_{i}^{\prime \prime F} \quad \forall i \in M_{F}$ & $\sum_{j \in N_{F}} x_{i j} \leq a_{i}^{\prime F} \quad \forall i \in M_{F}$ \\
\hline$\sum_{i \in M_{F}} x_{i j} \geq b_{j}^{\prime F} \quad \forall j \in N_{F}$ & $\sum_{i \in M_{F}} x_{i j} \geq b_{j}^{\prime \prime F} \quad \forall j \in N_{F}$ \\
\hline
\end{tabular}

where $X_{2}$ solves

$\left(P_{2}\right) \min _{X_{2}} Z_{2}\left(X_{1}, X_{2}\right)=\left(\sum_{i=1}^{M} \sum_{j=1}^{N}\left[p_{i j}^{\prime}, p_{i j}^{\prime \prime}\right] x_{i j}\right)\left(\sum_{i=1}^{M} \sum_{j=1}^{N}\left[q_{i j}^{\prime}, q_{i j}^{\prime \prime}\right] x_{i j}\right) \quad$ (for a given $\left.X_{1}\right)$

subject to

$$
\left.\begin{array}{l}
\sum_{j \in N_{F}} x_{i j} \leq\left[a_{i}^{\prime F}, a_{i}^{\prime \prime}\right] \forall i \in M_{F} \\
\sum_{i \in M_{F}} x_{i j} \geq\left[b_{j}^{\prime{ }^{F}}, b_{j}^{\prime \prime}\right] \forall j \in N_{F}
\end{array}\right\}
$$

with $x_{i j} \geq 0 \forall(i, j) \in M \times N$. Proceeding in the same manner as in BLFTP-NN, we will find individual best and worst solution at both the levels. For that, again we will follow Proposition 1 for the constraints. As the objective functions are indefinite quadratic, we proceed by taking appropriate coefficients as shown in Tables 10 and 11 .

As in BLFTP-NN, after finding the best and the worst optimal solution at both the levels, we will take target intervals of the $t$ th level objective function and preference bounds for the decision variables.

The proposed goal programming model to find the satisfactory solution is given by:

$$
\operatorname{Min} \sum_{t=1}^{2}\left(\bar{D}_{t}+\overline{\bar{D}}_{t}\right)
$$




$$
\begin{array}{r}
\text { subject to } \\
\bar{Z}_{t}+\bar{D}_{t}=Y_{t}^{* *}, \\
\overline{\bar{Z}}_{t}+\overline{\bar{D}}_{t}=-Y_{t}^{*}, \\
\sum_{j \in N_{L}} x_{i j} \leq a_{i}^{\prime \prime}, \quad \sum_{j \in N_{L}} x_{i j} \geq a_{i}^{\prime L} \forall i \in M_{L}, \\
\sum_{i \in M_{L}} x_{i j} \leq b_{j}^{\prime \prime}, \quad \sum_{i \in M_{L}} x_{i j} \geq b_{j}^{\prime}{ }^{L} \forall j \in N_{L}, \\
\sum_{j \in N_{F}} x_{i j} \leq a_{i}^{\prime \prime}, \quad \sum_{j \in N_{F}} x_{i j} \geq a_{i}^{\prime F} \forall i \in M_{F}, \\
\sum_{i \in M_{F}} x_{i j} \leq b_{j}^{\prime \prime}, \quad \sum_{i \in M_{F}} x_{i j} \geq b_{j}{ }^{F} \forall j \in N_{F}, \\
\left(x_{1}^{b}\right)_{i j}-\left(l_{1}\right)_{i j} \leq\left(x_{1}^{b}\right)_{i j} \leq\left(x_{1}^{b}\right)_{i j}+\left(u_{1}\right)_{i j}, i \in M^{L}, j \in N^{L}, \\
\left(x_{2}^{b}\right)_{i j}-\left(l_{2}\right)_{i j} \leq\left(x_{2}^{b}\right)_{i j} \leq\left(x_{2}^{b}\right)_{i j}+\left(u_{2}\right)_{i j}, i \in M^{F}, j \in N^{F}, \\
\bar{D}_{t}, \overline{\bar{D}}_{t}, X \geq 0, t=1,2 .
\end{array}
$$

\section{Numerical illustration of BLIQTP-NN}

\subsection{Problem definition}

The problem definition and assumptions are the same as in Section 6.1.

\subsection{Solved numerical (BLIQTP-NN)}

In this illustration, the leader's (vaccine manufacturer) objective function is indefinite quadratic $\&$ aims at minimizing the product of the neutrosophic transportation cost and neutrosophic depreciation cost when vaccines are transported from manufacturing units to distribution centres. The follower's (distributor) objective function is also indefinite quadratic and aims at minimizing the product of neutrosophic transportation cost and neutrosophic depreciation cost when vaccine travels from distribution centres to the health centres for administration.

At the upper level, let the manufacturing units (MU) have $4+3 I, 6+2 I, 5+3 I$ and $5+$ $2 I$ as their respective supply quantity. Let the distribution centres have $5+4 I, 3+3 I, 3+3 I$ and $5+4 I$ as their respective demands. At the lower level, let the health centres have $4+$ $2 I, 5+3 I, 6+2 I$ and $5+I$ as their respective demands. Here, we have taken a unbalanced transportation problem with total supply and total demands at upper level equal to $20+10 I$ and $16+14 I$ while the total supply \& total demands at lower level equal to $16+14 I$ and 20 $+8 I$.

Tables 12 and 13 represents the neutrosophic transportation cost and neutrosophic depreciation cost at upper and lower level, respectively. In each cell $(i, j)$ of both the tables, top entry denotes the neutrosophic transportation cost and bottom entry denotes neutrosophic depreciation cost when vaccine is transported from $i$ th source to $j$ th destination. The computing software LINGO 17.0 is used to solve the problem (BLIQTP-NN). 
Table 12 Upper level neutrosophic transportation problem

\begin{tabular}{lllll}
\hline MU/DC & Maharashtra & Tamil Nadu & Rajasthan & Haryana \\
\hline Ahmedabad & $3+3 I$ & $4+I$ & $2+2 I$ & $1+4 I \rightarrow\left[f_{i j}+\tilde{f}_{i j} I\right]$ \\
& $1+I$ & $1+2 I$ & $5+4 I$ & $5+3 I \rightarrow\left[d_{i j}+\tilde{d}_{i j} I\right]$ \\
Hyderabad & $4+2 I$ & $6+3 I$ & $7+2 I$ & $9+2 I$ \\
& $1+I$ & $7+3 I$ & $2+4 I$ & $2+3 I$ \\
Bengaluru & $4+3 I$ & $1+2 I$ & $3+2 I$ & $1+I$ \\
\multirow{2}{*}{ Delhi } & $8+I$ & $7+4 I$ & $3+2 I$ & $5+2 I$ \\
& $2+3 I$ & $3+4 I$ & $1+2 I$ & $5+4 I$ \\
& $1+3 I$ & $8+I$ & $5+2 I$ & $6+2 I$ \\
\hline
\end{tabular}

Table 13 Lower level neutrosophic transportation problem

\begin{tabular}{lllll}
\hline DC/HC & North region & East region & West region & South region \\
\hline Maharashtra & $1+3 I$ & $2+3 I$ & $3+3 I$ & $6+4 I \rightarrow\left[p_{i j}+\tilde{p}_{i j} I\right]$ \\
Tamil Nadu & $2+5 I$ & $1+3 I$ & $2+2 I$ & $7+4 I \rightarrow\left[q_{i j}+\tilde{q}_{i j} I\right]$ \\
& $1+6 I$ & $2+I$ & $4+I$ & $5+I$ \\
Rajasthan & $3+3 I$ & $2+I$ & $2+2 I$ & $3+2 I$ \\
& $3+2 I$ & $3+2 I$ & $3+3 I$ & $4+3 I$ \\
Haryana & $4+2 I$ & $4+4 I$ & $7+2 I$ & $2+3 I$ \\
& $3+4 I$ & $8+I$ & $1+3 I$ & $7+2 I$ \\
& $1+2 I$ & $2+4 I$ & $5+4 I$ & $2+2 I$
\end{tabular}

The source and demand constraints at upper level are:

$$
\begin{gathered}
\sum_{j=1}^{4} x_{1 j} \leq 4+3 I, \sum_{j=1}^{4} x_{2 j} \leq 6+2 I, \sum_{j=1}^{4} x_{3 j} \leq 5+3 I, \sum_{j=1}^{4} x_{4 j} \leq 5+2 I, \\
\sum_{i=1}^{4} x_{i 1} \geq 5+4 I, \sum_{i=1}^{4} x_{i 2} \geq 3+3 I, \sum_{i=1}^{4} x_{i 3} \geq 3+3 I, \sum_{i=1}^{4} x_{i 4} \geq 5+4 I .
\end{gathered}
$$

The source and demand constraints at lower level are:

$$
\begin{gathered}
\sum_{j=1}^{4} x_{1 j} \leq 5+4 I, \sum_{j=1}^{4} x_{2 j} \leq 3+3 I, \sum_{j=1}^{4} x_{3 j} \leq 3+3 I, \sum_{j=1}^{4} x_{4 j} \leq 5+4 I, \\
\sum_{i=1}^{4} x_{i 1} \geq 4+2 I, \sum_{i=1}^{4} x_{i 2} \geq 5+3 I, \sum_{i=1}^{4} x_{i 3} \geq 6+2 I, \sum_{i=1}^{4} x_{i 4} \geq 5+I .
\end{gathered}
$$

Let, $X_{1}=\left\{x_{11}, x_{21}, x_{31}, x_{41}\right\}$ be the variables controlled by the upper level problem and $X_{2}=\left\{x_{12}, x_{13}, x_{14}, x_{22}, x_{23}, x_{24}, x_{32}, x_{33}, x_{34}, x_{42}, x_{43}, x_{44}\right\}$ be the variables controlled by the lower level problem.

Proceeding as same in Sect. 6.2, by taking $\mathrm{I} \in[0,1]$, the problem reduces to a BLIQTP with interval coefficients. Tables 10 and 11 will be used to formulate the optimization problem for finding best and the worst solution for both levels. Appropriate dummy source and destination 
Table 14 Individual solutions

\begin{tabular}{lll}
\hline Solution & Best & Worst \\
\hline Upper Level & $\bar{Z}_{1}=1472$ & $\overline{\bar{Z}}_{1}=7395$ \\
& at $x_{1}^{b}=$ & at $x_{1}^{w}=$ \\
& $(0,3,0,4,0,0,0,0,0,0,1,1,5,0,2,0)$ & $(0,4,0,0,6,0,0,0,0,0,0,5,3,0,2,0)$ \\
\hline Solution & Best & Worst \\
\hline Lower Level & $\bar{Z}_{2}=1922$ & $\overline{\bar{Z}}_{2}=5280$ \\
& at $x_{2}^{b}=$ & at $x_{2}^{w}=$ \\
& $(0,5,4,0,0,0,2,0,0,0,0,5,4,0,0,0)$ & $(0,5,0,0,0,3,0,0,1,0,0,2,5,0,0,0)$ \\
\hline
\end{tabular}

are added to balance the transportation problem. The individual solutions are compiled in Table 14.

Let the target level of the leader's objective function be given by $\left[Y_{1}^{*}, Y_{1}^{* *}\right]=[1600,7000]$ and let the target level of the follower's objective function be $\left[Y_{2}^{*}, Y_{2}^{* *}\right]=[2200,5200]$.

The goal programming model developed along with the preference bounds on the decision variables is represented as

$$
\operatorname{Minimize}\left(\bar{D}_{1}+\bar{D}_{2}+\overline{\bar{D}}_{1}+\overline{\bar{D}}_{2}\right)
$$

subject to

$$
\begin{aligned}
& \left(3 x_{11}+4 x_{12}+2 x_{13}+x_{14}+4 x_{21}+6 x_{22}+7 x_{23}+9 x_{24}+4 x_{31}+x_{32}+3 x_{33}+x_{34}\right. \\
& \left.\quad+2 x_{41}+3 x_{42}+x_{43}+5 x_{44}\right) *\left(x_{11}+x_{12}+5 x_{13}+5 x_{14}+x_{21}+7 x_{22}+2 x_{23}+2 x_{24}\right. \\
& \left.+8 x_{31}+7 x_{32}+3 x_{33}+5 x_{34}+x_{41}+8 x_{42}+5 x_{43}+6 x_{44}\right)+\bar{D}_{1}=7000 \\
& \\
& \quad+6 x_{11}+5 x_{12}+4 x_{13}+5 x_{14}+6 x_{21}+9 x_{22}+9 x_{23}+11 x_{24}+7 x_{31}+3 x_{32}+5 x_{33} \\
& \left.\quad+2 x_{34}+5 x_{41}+7 x_{42}+3 x_{43}+9 x_{44}\right) *\left(2 x_{11}+3 x_{12}+9 x_{13}+8 x_{14}+2 x_{21}+10 x_{22}\right. \\
& \quad+6 x_{23}+5 x_{24}+9 x_{31}+11 x_{32}+5 x_{33}+7 x_{34}+4 x_{41}+9 x_{42}+7 x_{43} \\
& \left.\left.\quad+8 x_{44}\right)\right) *(-1)+\bar{D}_{1}=-1600
\end{aligned}
$$

$$
\begin{aligned}
& \left(x_{11}+2 x_{12}+3 x_{13}+6 x_{14}+x_{21}+2 x_{22}+4 x_{23}+5 x_{24}+3 x_{31}+3 x_{32}+3 x_{33}+4 x_{34}\right. \\
& \left.\quad+3 x_{41}+8 x_{42}+x_{43}+7 x_{44}\right) *\left(2 x_{11}+x_{12}+2 x_{13}+7 x_{14}+3 x_{21}+2 x_{22}+2 x_{23}+3 x_{24}\right. \\
& \left.\left.\quad+4 x_{31}+4 x_{32}+7 x_{33}+2 x_{34}+x_{41}+2 x_{42}+5 x_{43}+2 x_{44}\right)\right)+\bar{D}_{2}=5200, \\
& \left(4 x_{11}+5 x_{12}+6 x_{13}+10 x_{14}+7 x_{21}+3 x_{22}+5 x_{23}+6 x_{24}+5 x_{31}+5 x_{32}+6 x_{33}+7 x_{34}\right. \\
& \left.\quad+7 x_{41}+9 x_{42}+4 x_{43}+9 x_{44}\right) *\left(7 x_{11}+4 x_{12}+4 x_{13}+11 x_{14}+6 x_{21}+3 x_{22}+4 x_{23}\right. \\
& \left.\left.\quad+5 x_{24}+6 x_{31}+8 x_{32}+9 x_{33}+5 x_{34}+3 x_{41}+6 x_{42}+9 x_{43}+4 x_{44}\right)\right) *(-1) \\
& \quad+\bar{D}_{2}=-2200,
\end{aligned}
$$


Table 15 Satisfactory solution of BLIQTP-NN

\begin{tabular}{llc}
\hline Solution point & Objective values of leader & Objective values of follower \\
\hline$(0,4,1,0,0,1,5,0,0,0,0,5,5,0,0,0)$ & $(4144,13108)$ & $(2244,10292)$
\end{tabular}

$$
\begin{gathered}
x_{11}+x_{12}+x_{13}+x_{14} \leq 7, \quad x_{11}+x_{21}+x_{31}+x_{41} \leq 6, \\
x_{21}+x_{22}+x_{23}+x_{24} \leq 6, \quad x_{12}+x_{22}+x_{32}+x_{42} \leq 6, \\
x_{31}+x_{32}+x_{33}+x_{34} \leq 6, \quad x_{13}+x_{23}+x_{33}+x_{43} \leq 6, \\
x_{41}+x_{42}+x_{43}+x_{44} \leq 7, \quad x_{14}+x_{24}+x_{34}+x_{44} \leq 6, \\
x_{11}+x_{21}+x_{31}+x_{41} \geq 5, \quad x_{11}+x_{12}+x_{13}+x_{14} \geq 5, \\
x_{12}+x_{22}+x_{32}+x_{42} \geq 5, \quad x_{21}+x_{22}+x_{23}+x_{24} \geq 6, \\
x_{13}+x_{23}+x_{33}+x_{43} \geq 6, \quad x_{31}+x_{32}+x_{33}+x_{34} \geq 5, \\
x_{14}+x_{24}+x_{34}+x_{44} \geq 5, \quad x_{41}+x_{42}+x_{43}+x_{44} \geq 5, \\
0 \leq x_{11} \leq 0+5,5-4 \leq x_{12} \leq 5+5,4-3 \leq x_{13} \leq 4+3,0 \leq x_{14} \leq 0+5, \\
0 \leq x_{21} \leq 0+3,0 \leq x_{22} \leq 0+5,2-1 \leq x_{23} \leq 2+5,0 \leq x_{24} \leq 0+5, \\
0 \leq x_{31} \leq 0+2,0 \leq x_{32} \leq 0+5,0 \leq x_{33} \leq 0+5,5-5 \leq x_{34} \leq 5+5, \\
5-4 \leq x_{41} \leq 5+1,0 \leq x_{42} \leq 0+5,0 \leq x_{43} \leq 0+5,0 \leq x_{44} \leq 0+5, \\
x_{i j} \geq 0 \forall i, j, \bar{D}_{1}, \bar{D}_{2}, \overline{\bar{D}}_{1}, \overline{\bar{D}}_{2} \geq 0
\end{gathered}
$$

The solution of the proposed goal programming model in shown in Table 15 .

\section{Sensitivity analysis}

For different choices of the preference bounds of the upper and lower level decision variables, the solution points obtained by solving the proposed model are shown in Tables 16 and 17. 


\section{Flowchart for solving BLFTP-NN and BLIQTP-NN}

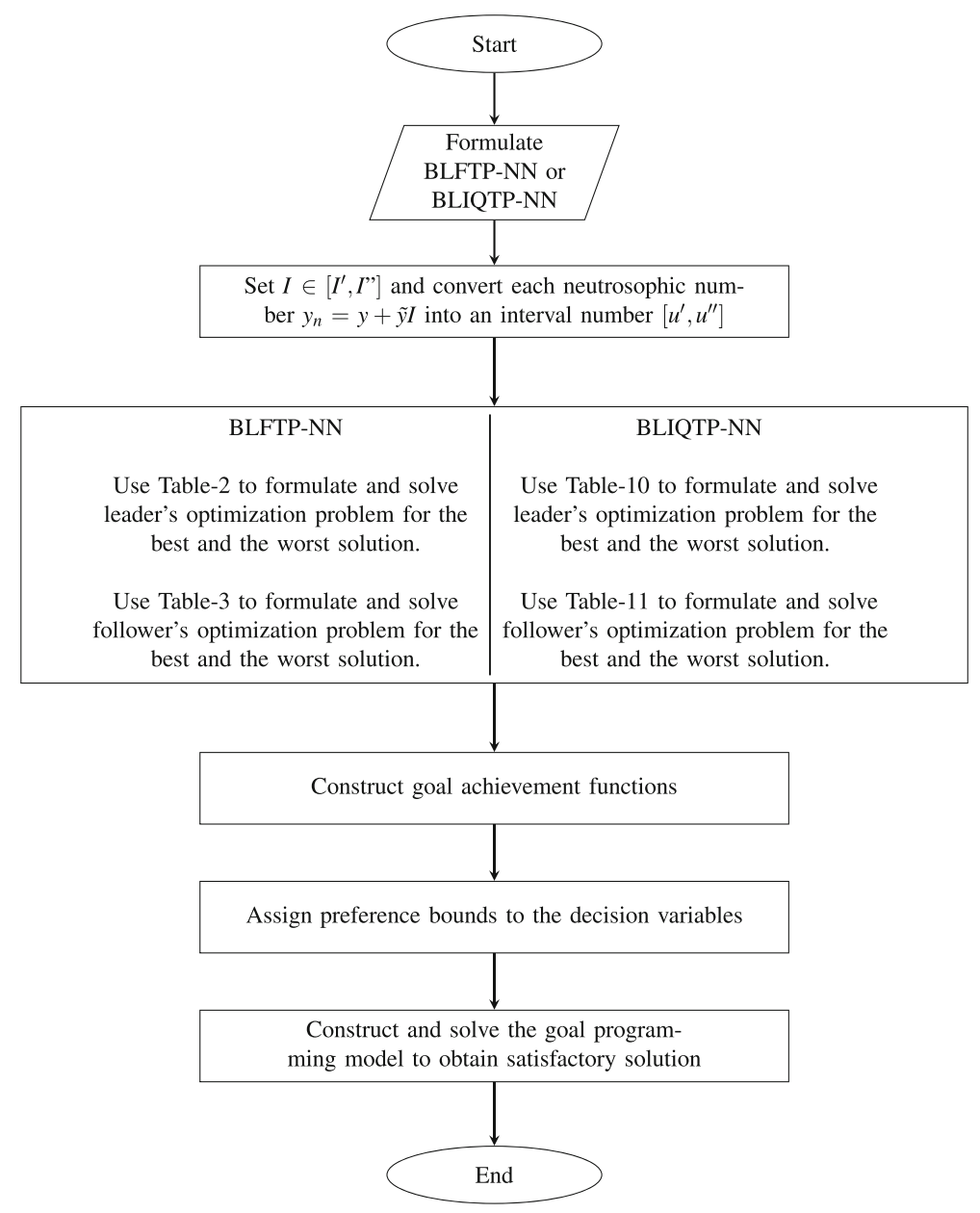

\section{Conclusion and future work}

We have tried to formulate the journey of a vaccine from its manufacture to delivery using bilevel transportation problem using two different objective functions viz. linear fractional and indefinite quadratic. Apart from LINGO, the reported problems have also been solved in MATHEMATICA and MATLAB and similar results were obtained.

The relevance of the paper is that it models the current issues of the transportation sector which is trying to strategically transport the vaccine whose supply, demand and transportation cost is indeterminate. Pandemic situations like COVID-19 need efficient and timely solutions of transportation sector with the cooperation of the decision makers as well. This will ultimately lead to a smooth conduction of the vaccination drive and the financial management will help in further research of vaccines and manpower management. 


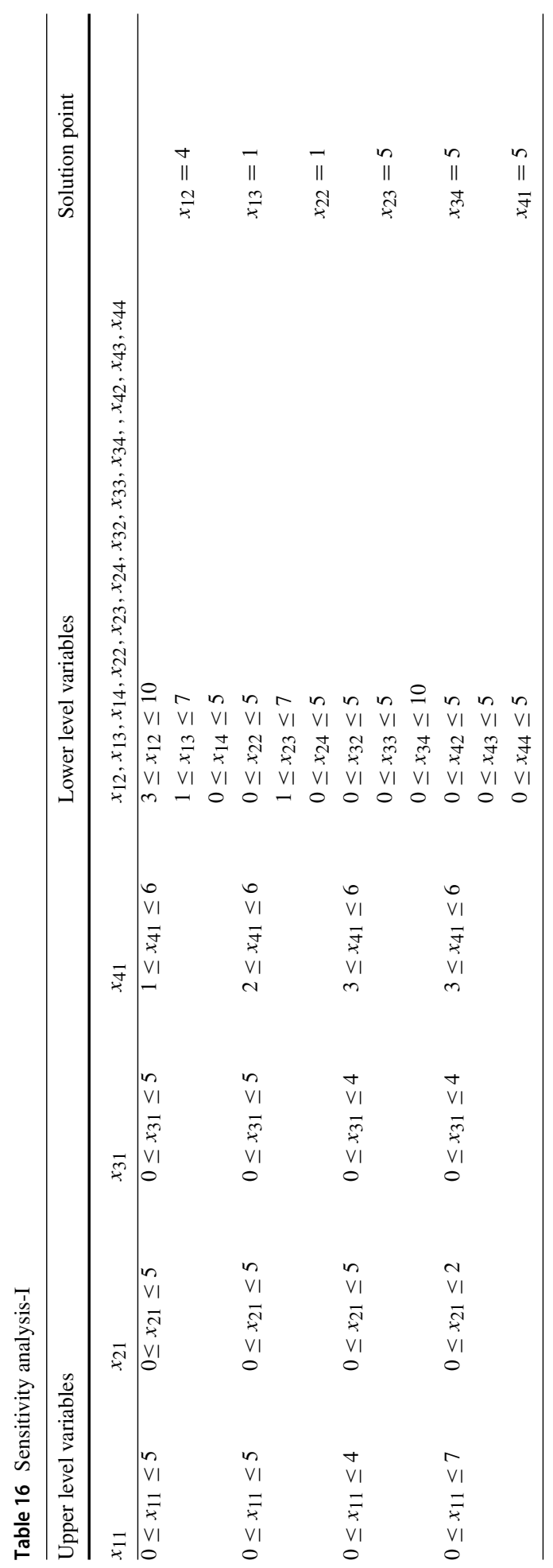




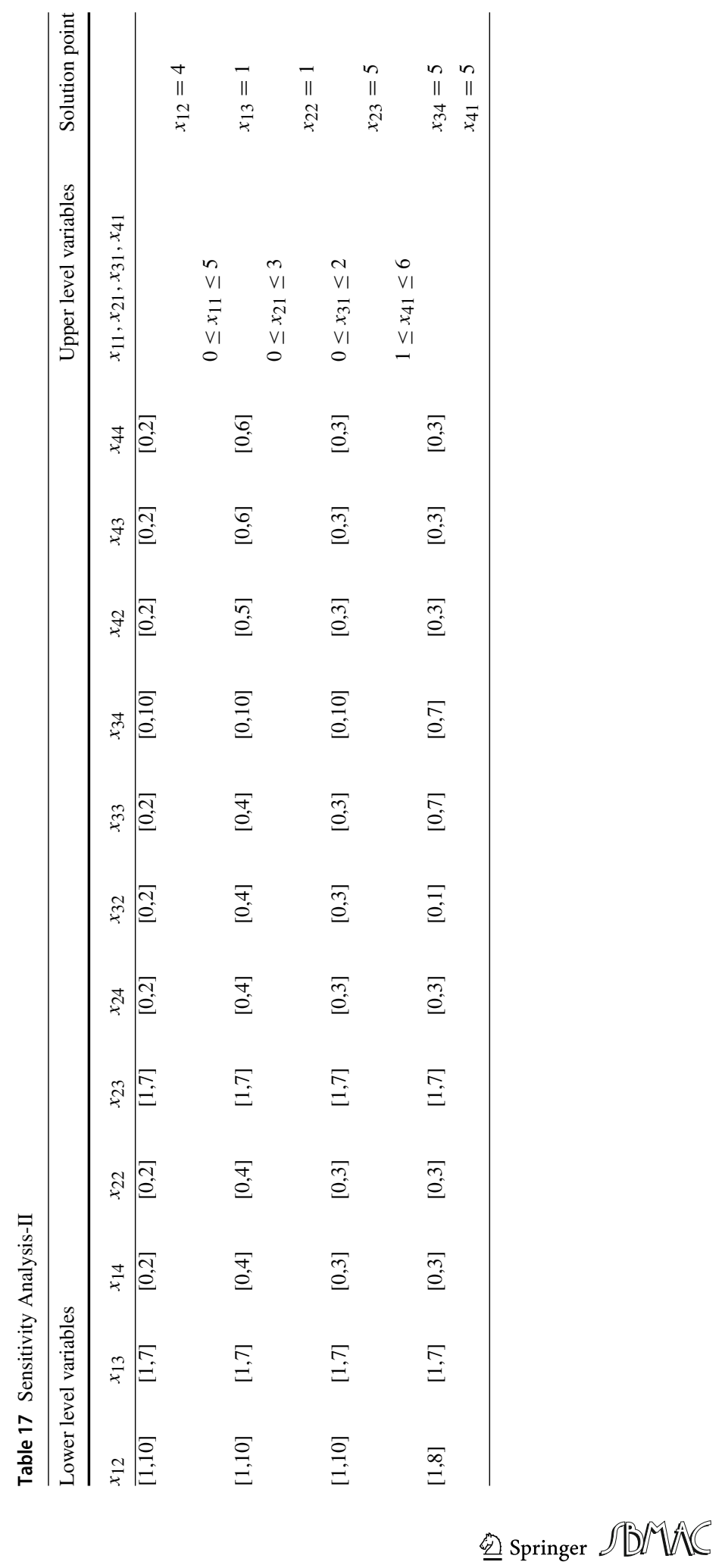


Till now there is no paper in the literature which deals with BLFTP-NN and BLIQTPNN with neutrosophic numbers of the form $P+Q I$. The problem can be extended to a trilevel transportation problem, multiobjective BLTP-NN and different interval programming methods can be employed to obtain a satisfactory solution.

Acknowledgements The authors are readily grateful to the reviewers and the associate editor for their valuable suggestions, incorporating which has helped us in improving the quality of the paper to a great extent.

Funding The authors did not receive support from any organization for the submitted work.

Availability of data and material The data used in the numerical illustrations are hypothetical.

\section{Declarations}

Conflict of interest The authors declare that they have no conflict of interest. The authors declare they have no financial or non-financial interests.

Code availability The reported problems have been solved in MATLAB, MATHEMATICA and LINGO and similar results were obtained.

\section{References}

Anithakumari T, Venkateswarlu B, Akilbasha A (2021) Optimizing a fully rough interval integer solid transportation problems. J Intell Fuzzy Syst 41(1):2429-2439

Arora R, Arora SR (2011) Solving linear-quadratic bilevel programming problem using Kuhn-Tucker conditions. Adv Model Optim 13(3):366-380

Arora R, Thirwani D (2013) Bilevel capacitated fixed charge transportation problem. Adv Model Optim 15(3):645-669

Bialas WF, Karwan MH (1982) On two level optimization. IEEE Trans Autom Control 27(1):211-214

Bialas W, Karwan M (1984) Two-level linear programming. Manag Sci 30:1004-1020

Bracken J, McGill J (1973) Mathematical programs with optimization problems in the constraints. Oper Res 21:37-44

Candler W, Townsley R (1982) A linear two-level programming problem. Comput Oper Res 9:59-76

Chakraborty A, Broumi S, Singh PK (2019) Some properties of pentagonal neutrosophic numbers and its applications in transportation problem environment. Neutrosophic Sets Syst 28(1):200-215

Chinneck JW, Ramadan K (2000) Linear programming with interval coefficients. Oper Res Soc 51:209-220

Das SK, Goswami A, Alam SS (1999) Multiobjective transportation problem with interval cost, source and destination parameters. Eur J Oper Res 117(1):100-112

Garg H, Rizk-Allah RM (2021) A novel approach for solving rough multi-objective transportation problem: development and prospects. Comput Appl Math 40(4):1-24

Kaushal B, Arora R, Arora S (2020) An aspect of bilevel fixed charge fractional transportation problem. Int J Appl Comput Math 6(1):1-9

Khandelwal A, Puri MC (2008) Bilevel time minimizing transportation problem. Discret Optim 5(4):714-723

Maiti I, Mandal T, Pramanik S (2019) Neutrosophic goal programming strategy for multi-level multi-objective linear programming problem. J Ambient Intell Hum Comput 2019:1-12

Midya S, Roy SK (2017) Analysis of interval programming in different environments and its application to fixed-charge transportation problem. Discrete Math Algorithms Appl 9(3):1750040

Mondal K, Pramanik S, Giri BC, Smarandache F (2018) NN-harmonic mean aggregation operatorsbased MCGDM strategy in a neutrosophic number environment. Axioms. https://doi.org/10.3390/ axioms 7010012

Narasimha PT, Jena PR, Majhi R (2021) Impact of COVID-19 on the Indian Seaport Transportation and maritime supply chain. Transp Policy 110:191-203

Paul N, Sarma D, Singh A, Bera UK (2020) A generalized neutrosophic solid transportation model with insufficient supply. Neutrosophic Sets Syst 35:177-187

Pramanik S, Dey PP (2019) Bi-level linear programming problem with neutrosophic numbers. Neutrosophic Sets Syst 21(1):110-121 
Pramanik S, Dey PP (2020) Multi-level linear programming problem with neutrosophic numbers: a goal programming strategy. Neutrosophic Sets Syst 29:242-254

Ramadan K (1996) Linear programming with interval coefficients. Doctoral dissertation, Carleton University

Rizk-Allah RM, Abo-Sinna MA (2021) A comparative study of two optimization approaches for solving bi-level multi-objective linear fractional programming problem. OPSEARCH 58(2):374-402

Safi MR, Razmjoo A (2013) Solving fixed charge transportation problem with interval parameters. Appl Math Model 37(18-19):8341-8347

Saini RK, Sangal A (2020) Application of single valued trapezoidal neutrosophic numbers in transportation problem. Neutrosophic Sets Syst 35:563-583

Shaocheng T (1994) Interval number and fuzzy number linear programming. Fuzzy Sets Syst 66(3):301-306

Sikkannan KP, Shanmugavel V (2020) Unraveling neutrosophic transportation problem using costs mean and complete contingency cost table. Neutrosophic Sets Syst 29(1):165-173

Singh A, Kumar A, Appadoo SS (2017) Modified approach for optimization of real life transportation problem in neutrosophic environment. Math Probl Eng 2017:1-9

Smarandache F (1998) Neutrosophy: neutrosophic probability, set, and logic. American Research Press, Rehoboth

Smarandache F (2013) Introduction to neutrosophic measure, neutrosophic integral, and neutrosophic probability. Sitech and Education Publisher, Columbus

Smarandache F (2014) Introduction to neutrosophic statistics. Sitech and Education Publishing, Columbus

Stackelberg H (1952) The theory of the market economy. Oxford University Press, New York

Sun X, Wandelt S, Zheng C, Zhang A (2021) COVID-19 pandemic and air transportation: successfully navigating the paper hurricane. J Air Transp Manag. https://doi.org/10.1016/j.jairtraman.2021.102062

Ye J (2016) Multiple-attribute group decision-making method under a neutrosophic number environment. J Intell Syst 25(3):377-386

Ye J (2018) Neutrosophic number linear programming method and its application under neutrosophic number environments. Soft Comput 22:4639-4646

Ye J, Cui W, Lu Z (2018) Neutrosophic number nonlinear programming problems and their general solution methods under neutrosophic number environments. Axioms. https://doi.org/10.3390/axioms7010013

Publisher's Note Springer Nature remains neutral with regard to jurisdictional claims in published maps and institutional affiliations. 